\title{
Somatostatin analog challenge test in the pre-surgical management of ACTH-secreting pheochromocytoma
}

\author{
Alessandro Rossini1 ${ }^{1}$ Francesca Perticone2 ${ }^{2}$ Laura Frosio ${ }^{2}$, Marco Schiavo Lena ${ }^{3}$ and \\ Roberto Lanzi²
}

'Endocrinology and Diabetes Unit, ASST Papa Giovanni XXIII, Bergamo, Italy, ${ }^{2}$ Endocrine Unit, Department of Internal Medicine, and ${ }^{3}$ Department of Pathology, San Raffaele Hospital, Milan, Italy
Correspondence should be addressed to F Perticone

Email

perticone.francesca@hsr.it

\section{Summary}

ACTH-secreting pheochromocytoma is a very rare cause of Cushing's syndrome, with a high morbidity and mortality risk due to both cortisol and catecholamines excess. We report the case of a 45 -year-old female patient with a $3 \mathrm{~cm}$, high-density, left adrenal mass, diagnosed as an ACTH-secreting pheochromocytoma. The biochemical sensitivity of the tumor to somatostatin analogues was tested by a $100 \mu \mathrm{g}$ s.c. octreotide administration, which led to an ACTH and cortisol reduction of 50 and $25 \%$ respectively. In addition to alpha and beta blockers, preoperative approach to laparoscopic adrenalectomy included octreotide, a somatostatin analogue, together with ketoconazole, in order to achieve an adequate pre-surgical control of cortisol release. Histopathological assessment confirmed an ACTH-secreting pheochromocytoma expressing type 2 and 5 somatostatin receptors (SSTR-2 and -5).

\section{Learning points:}

- ACTH-secreting pheochromocytomas represent a rare and severe condition, characterized by high morbidity and mortality risk.

- Surgical removal of the adrenal mass is the gold standard treatment, but adequate medical therapy is required preoperatively to improve the surgical outcome and to avoid major complications.

- Somatostatin analogs, in addition to other medications, may represent a useful therapeutic option for the presurgical management of selected patients.

- In this sense, the octreotide challenge test is a useful tool to predict favorable therapeutic response to the treatment.

\section{Background}

Cushing's syndrome (CS) is an uncommon disorder, with an incidence of $0.7-2.4$ cases per million per year (1). The majority of cases result from adrenocorticotropin (ACTH) hypersecretion, usually from a pituitary adenoma. In a smaller percentage of cases, ACTH is over-secreted from a non-pituitary source, such as bronchial carcinoid, smallcell lung carcinoma or thyroid medullary carcinoma (1). CS resulting from an ACTH-secreting pheochromocytoma is an even more rare and challenging condition that combines hypercortisolism and catecholamine excess, leading to severe and potentially life-threatening complications (2). About 60 cases have been reported in literature so far (2). The gold standard treatment of this condition is adrenalectomy, preceded by adequate medical preparation to obtain adequate control of hormonal hypersecretions. Here, we report a case of CS due to an 
ACTH-secreting pheochromocytoma and the beneficial effect of the combined ketoconazole-somatostatin analog treatment in the pre-surgical management of the patient.

\section{Case presentation}

A 45-year-old woman on oral hypoglycemic therapy (metformin $850 \mathrm{mg}$ b.i.d. and pioglitazone $15 \mathrm{mg}$ b.i.d.) due to a recent diagnosis of type 2 diabetes mellitus contacted the emergency department of our hospital complaining of a rapid and consistent worsening of glycemic control, together with asthenia, fever and weight loss. A few days before, she had been evaluated in the outpatient clinic of the Department of Cardiology for palpitations and was found to be hypertensive. There was no history of smoking, alcohol, drugs abuse and allergies. Family history for endocrine diseases was negative except for type 2 diabetes. Her weight was $60 \mathrm{~kg}$ and the height $167 \mathrm{~cm}$ (BMI, $21.5 \mathrm{~kg} / \mathrm{m}^{2}$ ). Clinical examination confirmed hypertension $(180 / 90 \mathrm{mmHg})$ with a pulse rate of 110 bpm and revealed an evident sarcopenia and dehydration. Laboratory tests showed leucocytosis (18.000) with raised polymorphs $(93.5 \%)$, hypokalemia $(2.73 \mathrm{mmol} / \mathrm{L})$, hyperglycemia (serum glucose $242 \mathrm{mg} / \mathrm{dL}$ ), and normal renal and liver functions. The patient received oral and i.v. potassium, amlodipine $5 \mathrm{mg}$ orally and s.c. insulin therapy. She was subsequently admitted to our unit.

\section{Investigation}

Results of the hormonal assessment are depicted in Table 1. Urinary and serum cortisol and plasma ACTH were markedly increased and dexamethasone $1 \mathrm{mg}$ overnight failed to suppress serum cortisol levels, consistently with a condition of ACTH-dependent Cushing's syndrome. Aldosterone/renin ratio was within the normal limits. Ancillary findings were secondary hypothyroidism, central hypogonadism, and low IGF-1 levels, as usually observed in cases of overt hypercortisolism.

Serum CRH stimulation test failed to significantly increase both serum cortisol and plasma ACTH levels (Table 1). Serum cortisol levels remained unchanged after high-dose (8 $\mathrm{mg})$ dexamethasoneovernight administration. Taken together, all these findings were consistent with a non-pituitary (ectopic) source of the ACTH secretion. Following a s.c. $100 \mu \mathrm{g}$ octreotide administration, a 50\% decrease in ACTH levels and 25\% decrease in cortisol levels were observed (Table 2). The octreotide challenge test was performed under the surveillance of an expert physician, with a close monitoring of vital parameters.
Table 1 Pre-operative hormonal assessment of the patient.

\begin{tabular}{l}
\hline Biochemical parameters \\
\hline Cortisol (nmol/L) \\
UFC (nmol/day) \\
ACTH (pmol/L) \\
TSH ( $\mu$ U/mL) \\
Free T4 (pmol/L) \\
Free T3 (pmol/L) \\
Estradiol (pmol/L) \\
LH (mU/mL) \\
IGF-1 (nmol/L) \\
Cortisol after DST \\
1 mg (nmol/L) \\
Cortisol after DST \\
8 mg (nmol/L) \\
24-h urine adrenaline \\
(nmol/day) \\
24-h urine noradrenaline \\
(nmol/day) \\
24-h urine metanephrine \\
(nmol/day) \\
24-h urine \\
normetanephrine \\
(nmol/day) \\
Chromogranin A (nmol/L) \\
NSE ( $\mu$ g/L) \\
CRH stimulation test \\
ACTH (pmol/L) \\
Baseline \\
Peak \\
Cortisol (nmol/L) \\
Baseline \\
Peak \\
\end{tabular}

\begin{tabular}{c}
\hline Values \\
\hline 2237 \\
$>34483$ \\
87.5 \\
0.17 \\
6.9 \\
1.0 \\
$<92$ \\
1.2 \\
8.5 \\
2300 \\
2364
\end{tabular}

\begin{tabular}{c} 
Normal range \\
\hline $138-717$ \\
$1931-8827$ \\
$2-11.5$ \\
$0.25-5$ \\
$12-22$ \\
$3-6$ \\
- \\
- \\
$12-32$
\end{tabular}$$
<50
$$

982

$-$

884

$9-122$

2807

$71-506$

1135

$<1622$

$<2130$

4.16

17.9

$0.5-3$

(1)

66
74

74

2265

2480

ACTH, adrenocorticotropic hormone; $\mathrm{CRH}$, corticotropin-releasing hormone; DST, dexamethasone; IGF-1, insulin like growth factor 1; LH, luteinizing hormone; NSE, neuron-specific enolase; TSH, thyroidstimulating hormone; UFC, urinary free cortisol.

A computed tomography (CT) of the thorax and abdomen identified a $3 \mathrm{~cm}$ left adrenal mass, showing features consistent with a non-adenoma (Fig. 1). Magnetic resonance imaging (MRI) of the pituitary gland was normal. Further hormonal investigation showed high concentrations of urinary catecholamines

Table 2 Results of the somatostatin challenge test.

\begin{tabular}{|c|c|c|}
\hline Time (min) & ACTH $(p m o l / L)$ & Cortisol $(\mathrm{nmol} / \mathrm{L})$ \\
\hline 0 & 92 & 2289 \\
\hline 60 & 52 & 1820 \\
\hline 120 & 51 & 1931 \\
\hline 180 & 61 & 1725 \\
\hline 240 & 39 & 2179 \\
\hline 300 & 54 & 1986 \\
\hline
\end{tabular}

ACTH, adrenocorticotrop-ic hormone. 


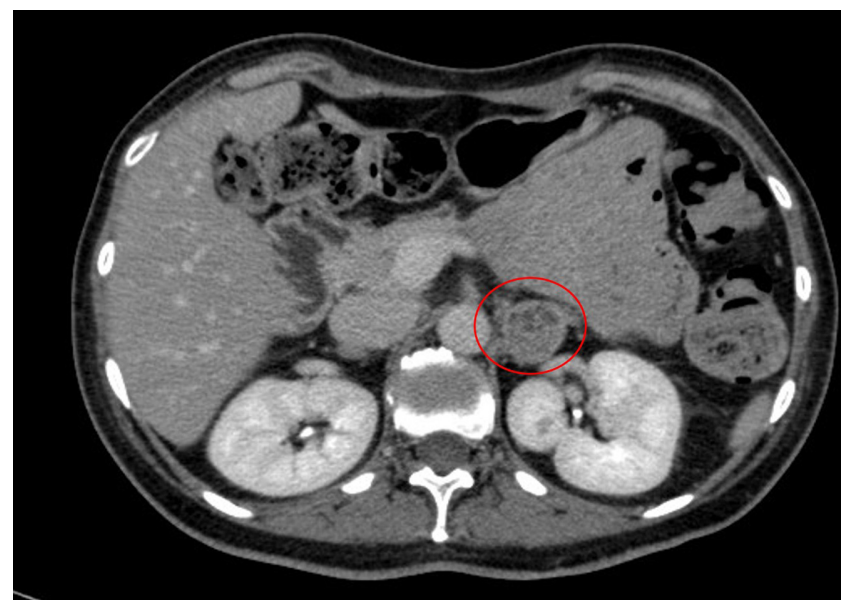

Figure 1

Coronal view at CT scan of the abdomen, showing a $30 \mathrm{~mm}$ high-density left adrenal mass.

and metanephrines, suggesting the presence of a pheochromocytoma. Chromogranin A (CGA) and neuronspecific enolase (NSE) were both elevated (Table 1).

\section{Treatment}

According to the whole picture, pre-operative management of the patient included alpha and beta blocker administration (doxazosin $8 \mathrm{mg}$ b.i.d. for 14 days, followed by doxazosin $8 \mathrm{mg}$ b.i.d. plus atenolol $50 \mathrm{mg}$ b.i.d.), ketoconazole (200 mg b.i.d.) and octreotide (100 $\mu$ g subcutaneously t.i.d.).

Preoperative biochemical controls showed a significant reduction of plasma ACTH $(204 \mathrm{pg} / \mathrm{mL})$ and serum cortisol $(46.6 \mu \mathrm{g} / \mathrm{dL})$ levels. An optimal control of blood pressure and pulse rate was achieved. Subsequently, the patient underwent a laparoscopic left adrenalectomy without any complication. Histopathological and immunohistochemical assessment confirmed an ACTHproducing pheochromocytoma expressing somatostatin receptors (SSTR) 2 and 5 (Fig. 2). The analysis of encoding ubiquitin-specific protease 8 (USP8) gene revealed no mutations. Postoperatively there was a rapid and complete remission of hypertension, hypokalemia and hyperglycemia. The patient discontinued all the hypoglycemic agents. Plasma ACTH showed a rapid and significant decrease to $5 \mathrm{pg} / \mathrm{mL} 3$ days after surgery. To avoid adrenal insufficiency due to the chronic suppression of the corticotropic axis, transient replacement therapy with hydrocortisone was introduced. There was a rapid recovery of the corticotropic axis that allowed the withdrawal of hydrocortisone after 15 days.

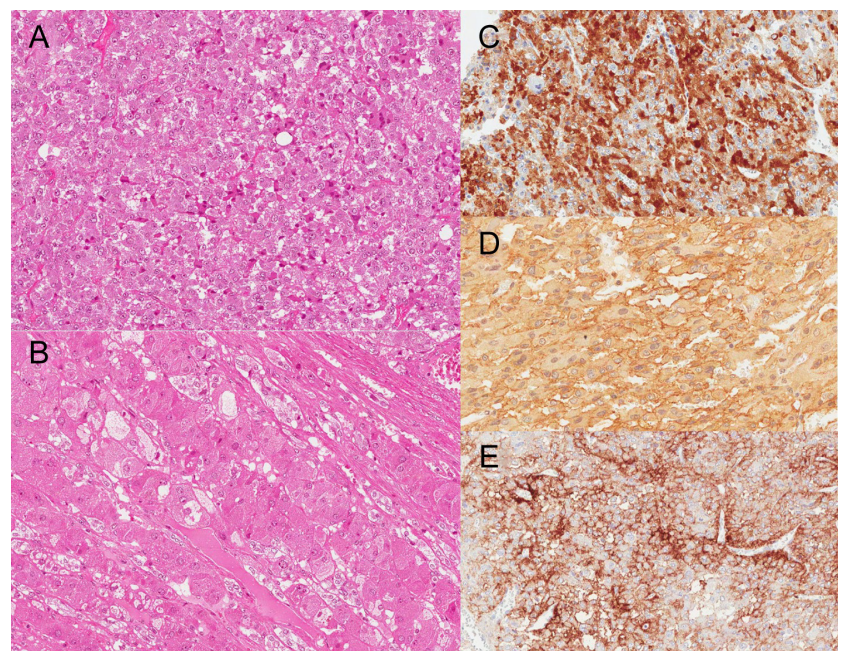

\section{Figure 2}

Histological specimens of the ACTH-secreting pheochromocytoma. (A) Hematoxylin and eosin stain of paraffin-embedded tumor tissue; (B) hematoxylin and eosin stain of hyperplastic adrenal cortex due to ACTH overproduction by the tumor; (C) ACTH immunohistochemistry of the adrenal tumor; (D) pheochromocytoma expression of somatostatin receptor 5 (SSTR5) assessed by specific antibodies; (E) pheochromocytoma cytoplasmatic and membranous expression of somatostatin receptor 2 (SSTR2) assessed by specific antibodies.

\section{Outcome and follow-up}

One month after adrenalectomy, cortisol suppression after dexamethasone $1 \mathrm{mg}$ overnight confirmed the complete resolution of endogenous hypercortisolism. In addition, urinary metanephrines normalized, $(105.3 \mu \mathrm{g} / 24 \mathrm{~h})$, and CGA decreased to normal values $(1.2 \mathrm{nmol} / \mathrm{L})$. At 4-year follow-up, there were not any biochemical, clinical or radiological sign of recurrence.

\section{Discussion}

ACTH-secreting pheochromocytoma represents a very rare and distinctive cause of CS, where clinical manifestations due to cortisol and catecholamines excess coexist. About 60 cases have been reported in literature so far (2). The syndrome predominantly occurs in women, with typical features including severe hypercortisolism, hypokalemia, rapidly worsening diabetes mellitus and hypertension, large adrenal tumor, and lack of typical phenotype of Cushing syndrome probably due to the fast development of hypercortisolism (2). Early diagnosis is often difficult, but is mandatory in order to avoid serious and potentially fatal complications and to guarantee an adequate pre- and post-surgical management. However, consensus regarding the optimal strategy regarding the perioperative clinical workup of such patients is still lacking, due to the rarity 
of the syndrome and the anecdotal reports presented in literature.

Here we report the efficacy of octreotide administration combined with ketoconazole, doxazosin and atenolol in the management of a 45-year woman with an ACTHsecreting pheochromocytoma, allowing an adequate presurgical control of cortisol and catecholamines excess.

Expression of SSTRs has been documented in pheochromocytomas (3), but clinical reports on the efficacy of somatostatin analogs published so far have yielded conflicting results $(4,5)$, due, at least in part, to the wide heterogeneity of SSTRs expression in the tumor and to their different affinity to the drugs (6). Identification of specific parameters predicting a favorable response to therapy is therefore crucial to select patients who may benefit from somatostatin analogs.

Ruggeri et al. (6) reported the case of a patient harboring a CRH-secreting pheochromocytoma, successfully treated with octreotide based on a positive octreotide scintigraphy. In that case, immunohistochemistry revealed the expression of SSTR1, SSTR2A, and SSTR2B receptor subtypes on tumor cells. However, Uwaifo et al. (7) described three cases of ectopic ACTH-dependent CS where pentetreotide scan failed to predict the therapeutic response to somatostatin analogs, with one patient even developing a paradoxical increase in ACTH and cortisol levels after a single octreotide administration. Similar observations have also been reported by other authors (8). Uwaifo et al. (7) suggested performing an octreotide challenge test to predict the ACTH and cortisol response to therapy but, to our knowledge, no reports are available in the literature on this topic in patients with ACTHsecreting pheochromocytoma.

To date, there is no established protocol to acutely test the hormonal sensitivity to somatostatin analogs in CS. We decided to perform a single acute challenge with $100 \mu \mathrm{g}$ octreotide, similarly to what has been described in acromegaly (9). The significant reduction of circulating ACTH levels prompted us to associate octreotide to ketoconazole in the pre-surgical workup of the patient, that in a few days led to a further reduction of ACTH together with cortisol levels. The combination of the two drugs with doxazosin and atenolol allowed an optimal pre- and intra-operative control of blood pressure, pulse rate, glycemic profile and electrolyte homeostasis.

In this setting, the effects of octreotide cannot be isolated from those of the associated drugs. Some authors, however, reported that ketoconazole alone may be unable to adequately control hypercortisolism in ACTH-secreting pheochromocytoma (10), exposing the patients to the wellknown perioperative complication due to cortisol excess.

\section{Declaration of interest}

The authors declare that there is no conflict of interest that could be perceived as prejudicing the impartiality of this case report.

\section{Funding}

This research did not receive any specific grant from any funding agency in the public, commercial or not-for-profit sector.

\section{Patient consent}

Written informed consent was obtained from the patient for publication of the submitted article and accompanying images.

\section{Author contribution statement}

A R and F P wrote this case report. L F was involved in clinical disease diagnosis and management. M S L was the referee for histological diagnosis and immunohistochemical studies. $\mathrm{R} L$ was the patient's attending physician and supervised the manuscript writing and revision.

\section{References}

1 Newell-Price J, Bertagna X, Grossman AB \& Nieman LK. Cushing's syndrome. Lancet 2006367 1605-1617. (https://doi.org/10.1016/ S0140-6736(06)68699-6)

2 Gabi JN, Milhem MM, Tovar YE, Karem ES, Gabi AY \& Khthir RA. Severe Cushing syndrome due to an ACTH-producing pheochromocytoma: a case presentation and review of the literature. Journal of the Endocrine Society 20182 621-630. (https://doi. org/10.1210/js.2018-00086)

3 De Herder WW \& Hofland LJ. Somatostatin receptors in pheochromocytoma. Frontiers of Hormone Research 200431 145-154. (https://doi.org/10.1159/000074662)

4 Lamarre-Cliche M, Gimenez-Roqueplo AP, Billaud E, Baudin E, Luton JP \& Plouin PF. Effects of slow-release octreotide on urinary metanephrine excretion and plasma chromogranin A and catecholamine levels in patients with malignant or recurrent phaeochromocytoma. Clinical Endocrinology 200257 629-634. (https://doi.org/10.1046/j.1365-2265.2002.01658.x)

5 Plouin PF, Bertherat J, Chatellier G, Billaud E, Azizi M, Grouzmann E \& Epelbaum J. Short-term effects of octreotide on blood pressure and plasma catecholamines and neuropeptide Y levels in patients with phaeochromocytoma: a placebo-controlled trial. Clinical Endocrinology 199542 289-294. (https://doi. $\operatorname{org} / 10.1111 /$ j.1365-2265.1995.tb01877.x)

6 Ruggeri RM, Ferraù F, Campennì A, Simone A, Barresi V, Giuffrè G, Tuccari G, Baldari S \& Trimarchi F. Immunohistochemical localization and functional characterization of somatostatin receptor subtypes in a corticotropin releasing hormone-secreting adrenal phaeochromocytoma: review of the literature and report of a case. European Journal of Histochemistry 200953 1-6. (https://doi. org/10.4081/ejh.2009.e1)

7 Uwaifo GI, Koch CA, Hirshberg B, Chen CC, Hartzband P, Nieman LK \& Pacak K. Is there a therapeutic role for octreotide in 
patients with ectopic Cushing's syndrome? Journal of Endocrinological Investigation 200326 710-717. (https://doi.org/10.1007/BF03347351)

8 Biagetti B, Simó-Servat O, Ortiz A, Iglesias C \& Mesa J. Ectopic Cushing's syndrome: paradoxical effect of somatostatin analogs. Endocrinología, Diabetes y Nutrición 201764 276-278. (https://doi org/10.1016/j.endinu.2017.01.004)

9 Karavitaki N, Botusan I, Radian S, Coculescu M, Turner HE \& Wass JA. The value of an acute octreotide suppression test in predicting long-term responses to depot somatostatin analogues in patients with active acromegaly. Clinical Endocrinology 200562 282-288. (https://doi.org/10.1111/j.1365-2265.2004.02191.x)

10 Flynn E, Baqar S, Liu D, Ekinci EI, Farrell S, Zajac JD, De Luise M $\&$ Seeman E. Bowel perforation complicating an ACTH-secreting phaeochromocytoma. Endocrinology, Diabetes \& Metabolism Case Reports 20162016 16-0061. (https://doi.org/10.1530/EDM16-0061)

Received in final form 31 October 2019

Accepted 6 November 2019 